\title{
Predictive factors for remission and recurrence in Cushing's \\ disease: a single-center study
}

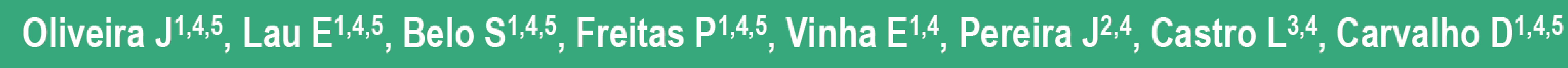

1Endocrinology, Diabetes and Metabolism Department; ${ }^{2}$ Neurosurgery Department; ${ }^{3}$ Pathological Anatomy Department; ${ }^{4}$ Centro Hospitalar São João, Porto, Portugal;

${ }^{5}$ Faculty of Medicine of University of Porto, Portugal

\section{Introduction}

Cushing's disease (CD) is characterized by pathologic hypercortisolism caused by an adrenocorticotropic hormone (ACTH)-secreting pituitary adenoma. The primary modality for definitive treatment is pituitary surgery. The rarity of $C D$ has made it difficult to establish reliable predictive factors of outcomes.

\section{Objective}

Assessment of clinical, hormonal, radiological, surgical and histological findings as predictors of remission and relapse of CD.

\section{Methods}

Cross-sectional, retrospective study of patients with CD who underwent pituitary surgery between January/1998-October/2013.

$\checkmark$ Serum cortisol (normal range: $7-25 \mu \mathrm{g} / \mathrm{dL}$ ), ACTH (normal range: $<63.3 \mathrm{ng} / \mathrm{L}$ ), urinary free cortisol (normal range: $75-270 \mu g / 24 \mathrm{~h}$ ).

\section{Results}

\section{5 patients After pituitary surgery $\left(1^{\text {st }}\right.$ or $2^{\text {nd }}$ intervention):}

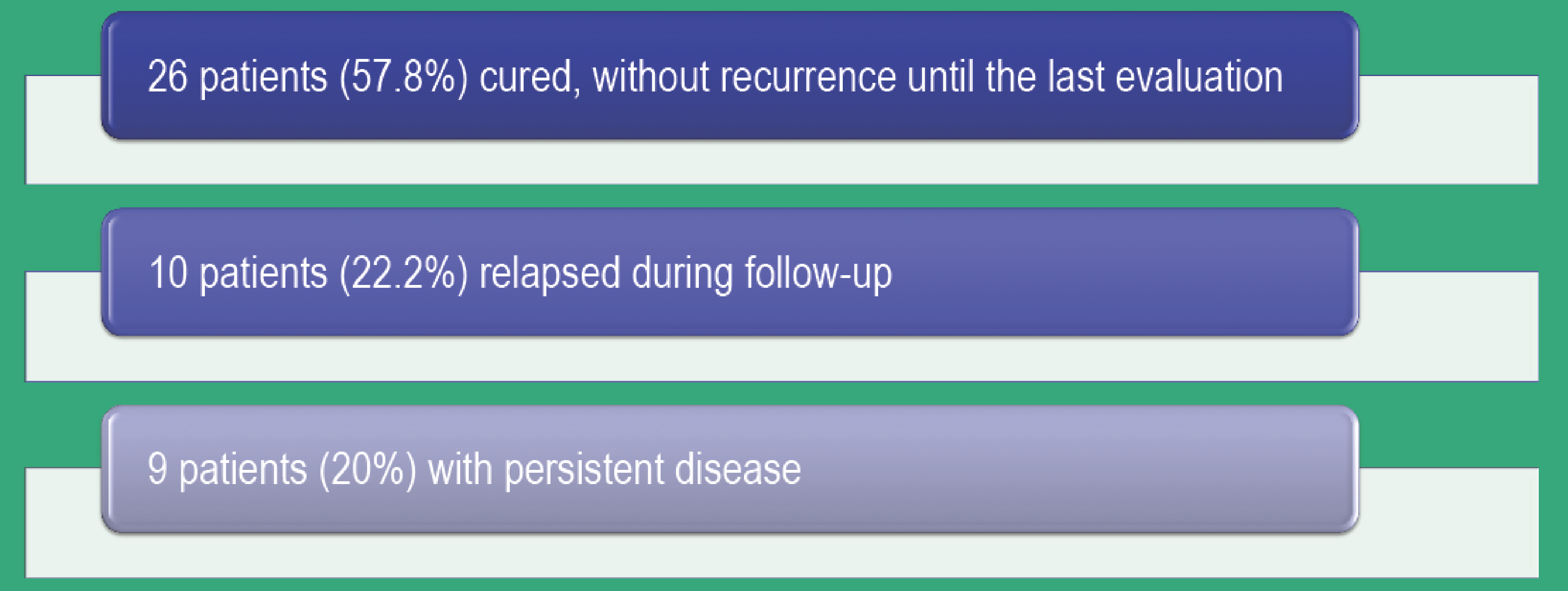

\begin{tabular}{|c|c|c|c|}
\hline & CURED & NOT CURED & RELAPSE \\
\hline Age [years, mean (SD)] & $37.8(12.6)$ & $42.2(14.3)$ & $35.5(13.1)$ \\
\hline \multicolumn{4}{|l|}{ Sex [n (\%)] } \\
\hline Male & $4(15.4 \%)$ & $2(22.2 \%)$ & - \\
\hline Female & $22(84.6 \%)$ & $7(77.8 \%)$ & $10(100 \%)$ \\
\hline
\end{tabular}

PreOPERATIVE COMORBIDITIES

\begin{tabular}{lccc} 
& CURED & NOT CURED & RELAPSE \\
\hline Arterial hypertension [n (\%)] & $15(57.7 \%)$ & $7(77.8 \%)$ & $7(70 \%)$ \\
Diabetes mellitus [n $(\%)]$ & $9(34.6 \%)$ & $1(11.1 \%)$ & $3(30 \%)$ \\
Dyslipidemia [n $(\%)]$ & $12(46.1 \%)$ & $4(44.4 \%)$ & $3(30 \%)$ \\
Psychiatric disorders [n (\%)] & $12(46.1 \%)$ & $3(33.3 \%)$ & $7(70 \%)$ \\
\hline
\end{tabular}

MAGNETIC RESONANCE IMAGING

\begin{tabular}{lccc} 
& CURED & NOT CURED & RELAPSE \\
\hline Microadenoma [n (\%)] & $15(60 \%)$ & $3(37,5 \%)$ & $5(50 \%)$ \\
Macroadenoma [n (\%)] & $6(24 \%)$ & $2(25 \%)$ & $1(10 \%)$ \\
Indirect signs of tumoral lesion [n (\%)] & - & $2(25 \%)$ & $1(10 \%)$ \\
No image [n (\%)] & $4(16 \%)$ & $1(12.5 \%)$ & $3(30 \%)$ \\
Total & $25(100 \%)$ & $8(100 \%)$ & $10(100 \%)$ \\
\hline
\end{tabular}

\section{BASAL HORMONAL StUdY}

\begin{tabular}{|c|c|c|c|}
\hline & CURED & NOT CURED & RELAPSE \\
\hline ACTH [ng/L, mean (SD)] & $72.5(38.8) n=26$ & $86.1(48.9) n=10$ & $75.0(54.8) n=8$ \\
\hline Serum cortisol [ $[\mu \mathrm{g} / \mathrm{dL}$, mean (SD] & $27.0(9.5) \mathrm{n}=26$ & $28.7(14.7) n=10$ & $30.9(7.0) n=7$ \\
\hline Urinary free cortisol [Mg/dia, mean (SD)] & $416.6(389.8) n=26$ & $575.6(451.9) \mathrm{n}=10$ & $540.4(535.9) n=8$ \\
\hline Overnight dexametasone supression test [ $\mu \mathrm{g} / \mathrm{dL}$, mean (SD)] & $24.8(17.1) n=16$ & $16.1(4.8) n=5$ & $37.7(25.9) n=4$ \\
\hline
\end{tabular}

\begin{tabular}{lccc}
\hline & CURED & NOT CURED & RELAPSE \\
\hline Transesphenoidal $[n(\%)]$ & $24(\%)$ & $9(\%)$ & $10(\%)$ \\
Transfrontal $[n(\%)]$ & $2(\%)$ & - & - \\
Total & $26(100 \%)$ & $9(100 \%)$ & $10(100 \%)$ \\
\hline
\end{tabular}

No significant differences between groups (cured, not cured, disease relapse) relating age, gender, presence of preoperative comorbidities (hypertension, DM, dyslipidemia and psychiatric disorders), basal hormonal study, magnetic resonance imaging findings or surgical technique.

\begin{tabular}{|c|c|c|c|c|}
\hline & CURED & NOT CURED & RELAPSE & $P$ \\
\hline ACTH-producing adenoma [n $(\%)]$ & $24(92.3 \%)$ & $5(55.6 \%)$ & $8(80 \%)$ & \\
\hline No adenoma identified [n (\%)] & $2(7.7 \%)$ & $4(44.4 \%)$ & $2(20 \%)$ & $<0.05^{*}$ \\
\hline Total & $26(100 \%)$ & $9(100 \%)$ & $10(100 \%)$ & \\
\hline
\end{tabular}

\section{Conclusion}

The absence of adenoma identification in pituitary histology after surgery was associated with higher risk of persistence or recurrence of $C D$. Those patients would benefit from a closer follow-up and early evaluation. No other predictive factors were found. 